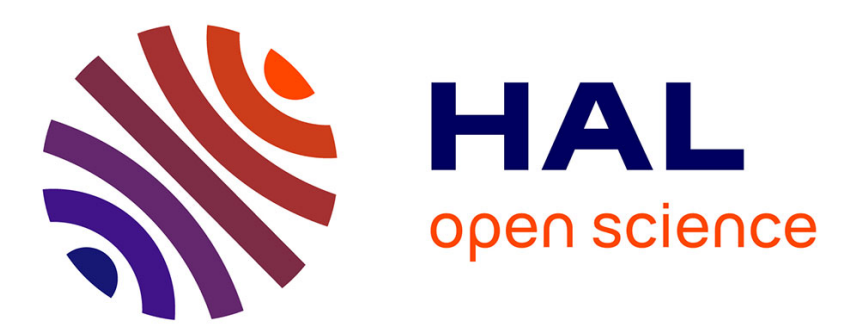

\title{
A Polytopic Proportional Integral Observer Design for Fault Diagnosis
}

B Rabaoui, H Hamdi, Mickael Rodrigues, N Benhadj Braiek

\section{To cite this version:}

B Rabaoui, H Hamdi, Mickael Rodrigues, N Benhadj Braiek. A Polytopic Proportional Integral Observer Design for Fault Diagnosis. INTERNATIONAL CONFERENCE ON ADVANCED SYSTEMS AND ELECTRICAL TECHNOLOGIES, Jan 2017, Hammamet, Tunisia. pp.14 - 17. hal-01413062

\section{HAL Id: hal-01413062 \\ https://hal.science/hal-01413062}

Submitted on 9 Dec 2016

HAL is a multi-disciplinary open access archive for the deposit and dissemination of scientific research documents, whether they are published or not. The documents may come from teaching and research institutions in France or abroad, or from public or private research centers.
L'archive ouverte pluridisciplinaire HAL, est destinée au dépôt et à la diffusion de documents scientifiques de niveau recherche, publiés ou non, émanant des établissements d'enseignement et de recherche français ou étrangers, des laboratoires publics ou privés. 


\title{
A Polytopic Proportional Integral Observer Design for Fault Diagnosis
}

\author{
B. Rabaoui, H. Hamdi, M. Rodriguesand N. BenHadj Braiek
}

\begin{abstract}
This paper deals with the problem of actuators faults detection and estimation for linear parameters variant (LPV) systems described by the polytopic representation. A Polytopic Proportional Integral Observer (PPIO) is designed to detect faults by generation of residuals signals and estimate system states and the applied faults. The (PPIO) gains are obtained by solving a set of linear matrices inequalities (LMIs). Performances of the proposed method are shown through a Van de Vusse reactor.
\end{abstract}

Index Terms-LPV system, Polytopic Proportional Integral Observer, Fault Detection and estimation, residual generation, LMIs.

\section{INTRODUCTION}

$\mathbf{I}$ $\mathrm{T}$ is well recognized that many practical dynamical systems are subject to various environmental changes, unknown disturbances, and changing operating conditions, thus sensors, actuators or components failure and faults in those systems are inevitable. Since any faults in a dynamical system may lead to significant performance degradation, serious system damages, and even loss of human life, it is essential to be able to detect and identify those faults in a timely manner so that necessary protective measures can be taken in advance. To that end, fault diagnosis of dynamic systems has received much attention and significant progress has been made in recent years [6].

A little while back, some research works have appeared that consider Linear Parameter Varying (LPV) modeling of the system performance to take into account wider and more rapid parameters variations. Such models can be used efficiently to represent some nonlinear systems.

Subsequently, much attention has been devoted to the development of robust fault-detection methods under external disturbances for LPV polytopic systems. Indeed, in [2], [4], [6], [8], [9], [11] and [15] the authors dealt the problem of fault diagnosis for LPV descriptor systems, multi-models descriptor systems, descriptor systems described by Takagi Sugeno approach and uncertain LPV systems in the continuous time. The problem of fault diagnosis is treated also in the discrete time in [1],[15], [18], [20] and [21].

Others papers as [3], [10], [11], [14], [16], [19] and [22] that were dedicated to states and faults estimation.

Some works are dedicated to the design of observers like design of full and reduced order observers [5], [13] and design of unknown input proportional multiple integral observer [14], ...

N. BenHadj Braiek, H. Hamdi and B. Rabaaoui are respectively the director and members of Advanced Systems Laboratory, Tunisia Polytechnic School, La Marsa 2078, Tunisia, e-mail: (rabaaouibouali@gmail.com).

***************************

Manuscript received Month Day, Year; revised Month Day, Year.
In this paper an approach based on residual generation and fault estimation is considered for LPV systems that can be modeled within a LPV polytopic setting. The proposed method is based on polytopic proportional integral observer (PPIO). This paper is organized as follows. The structures of the LPV systems and of the polytopic proportional integral observer (PPIO) are formulated in section II. The section III is dedicated to study the fault diagnosis. Finally, Section IV presents an application of obtained results on a Van de Vusse reactor, and Section $\mathrm{V}$ gives some concluding remarks.

\section{Problem Statement}

Let consider the following LPV system which is modeled by the following state space representation:

$$
\left\{\begin{array}{l}
\dot{x}(t)=A(\theta(t)) x(t)+B(\theta(t)) u(t) \\
y(t)=C x(t)
\end{array}\right.
$$

where $x(t) \in \Re^{n}, u(t) \in \Re^{p}$ and $y(t) \in \Re^{m}$ are respectively the state vector, the input vector and the measured output vector. $A(\theta(t)), B(\theta(t))$ and $C$ are continuous functions which depend on time varying parameter vector $\theta(t) \in \Re^{q}$. This vector is bounded and lies into a hypercube such that:

$$
\theta(t) \in \Gamma=\left\{\theta: \theta_{\min }(t) \leq \theta(t) \leq \theta_{\max }(t) ; \forall t \geq 0\right\}
$$

By assuming an affine dependance of the parameter vector $\theta(t)$ [8], [18], the system (1) can be described by a polytopic form, where it can be transformed into a convex combination of the vertices of $\Gamma$ such that:

$$
\left\{\begin{array}{l}
\dot{x}(t)=\sum_{i=1}^{h} \rho_{i}(\theta(t))\left[A_{i} x(t)+B_{i} u(t)\right] \\
y(t)=C x(t)
\end{array}\right.
$$

where $\rho(\theta(t))$ vary into the convex set $\Omega$.

$$
\begin{gathered}
\Omega=\left\{\rho(\theta(t)) \in R^{h}, \rho(\theta(t))=\left[\rho_{1}(\theta(t)), \ldots, \rho_{h}(\theta(t))\right]^{T} ;\right. \\
\rho_{i}(\theta(t)) \geq 0 \text { and } \sum_{i=1}^{h} \rho_{i}(\theta(t))=1
\end{gathered}
$$

The matrices $A_{i} \in \Re^{n \times n}, B_{i} \in \Re^{n \times p}$ and $C \in \Re^{m \times n}$ are time invariant for the $i^{\text {th }}$ model represented by a linear form. Let us consider the polytopic LPV system (3) affected by an additive actuator faults such that:

$$
\left\{\begin{array}{l}
\dot{x}(t)=\sum_{i=1}^{h} \rho_{i}(\theta(t))\left[A_{i} x(t)+B_{i} u(t)+F_{i} f(t)\right] \\
y(t)=C x(t)
\end{array}\right.
$$

where $f(t) \in \Re^{q}$ and $F_{i} \in \Re^{n \times q}$ represent respectively the fault vector and the faut distribution matrix. 
In this paper, the main objective is to design a Polytopic Proportional Integral Observer (PPIO) in order to estimate both the actuator faults and the states vector. The proposed (PPIO) can be described by the following structure [8]:

$$
\left\{\begin{aligned}
\dot{z}(t) & =\sum_{i=1}^{h} \rho_{i}(\theta(t))\left[N_{i} z(t)+G_{i} u(t)+L_{i} y(t)+H_{i} \hat{f}(t)\right] \\
\hat{x}(t) & =z(t)+M_{2} y(t) \\
\hat{y}(t) & =C \hat{x}(t) \\
\hat{\hat{f}}(t) & =\sum_{i=1}^{h} \rho_{i}(\theta(t)) \phi_{i}(y(t)-\hat{y}(t))
\end{aligned}\right.
$$

where $z(t) \in \Re^{n}, \hat{x}(t) \in \Re^{n}, \hat{y}(t) \in \Re^{m}$ and $\hat{f}(t) \in \Re^{q}$ are respectively the observer state vector, the estimated state vector, the estimated output vector and the the estimated actuator fault of $f(t)$.

$N_{i}, G_{i}, L_{i}, H_{i}, \phi_{i}$ and $M_{2}$ are unknown matrices with appropriate dimensions to be determined in following section.

\section{FAULT DIAGNOSIS}

\section{A. Polytopic Proportional Integral Observer (PPIO) Design}

Firstly, we consider the following assumptions:

Assumption A1: The matrix $C$ is of full row rank, ie:

$$
\operatorname{rank}(C)=m
$$

Assumption A2: The polytopic LPV system (5) is observable, ie:

$$
\operatorname{rank}\left(\begin{array}{c}
C \\
C A_{i} \\
\vdots \\
C A_{i}{ }^{n-1}
\end{array}\right)=n ; \forall i=1, \cdots, h
$$

Assumption A3: The polytopic LPV system (5)is detectable, ie:

$$
\operatorname{rank}\left(\begin{array}{c}
s I_{n}-A_{i} \\
C
\end{array}\right)=n ; \forall i=1, \cdots, h \text { where } s \in C
$$

In the following, the observer design requires the study of the state estimation error $e(t)$ which is defined such that:

$$
e(t)=x(t)-\hat{x}(t)
$$

We assume that there exist $M_{1} \in \Re^{n \times n}$ and $M_{2} \in \Re^{n \times m}$ which verify the following equation:

$$
M_{1}+M_{2} C=I_{n}
$$

Therefore the above equation can be rewritten as:

$$
\left[\begin{array}{ll}
M_{1} & M_{2}
\end{array}\right]\left[\begin{array}{l}
I_{n} \\
C
\end{array}\right]=I_{n}
$$

Thus, by using (6) and (11), the equation (10) will be rewritten as:

$$
\begin{aligned}
e(t) & =\left(I_{n}-M_{2} C\right) x(t)-z(t) \\
& =M_{1} x(t)-z(t)
\end{aligned}
$$

The dynamic estimation error is then described as follows:

$$
\begin{aligned}
\dot{e}(t) & =M_{1} \dot{x}(t)-\dot{z}(t) \\
& =\sum_{i=1}^{h} \rho_{i}(\theta(t))\left[\left(M_{1} A_{i}-L_{i} C-N_{i}+N_{i} M_{2} C\right) x(t)\right. \\
& +\left(M_{1} B_{i}-G_{i}\right) u(t)+\left(M_{1} F_{i}-H_{i}\right) f(t)+N_{i} e(t) \\
& \left.+H_{i} e_{f}(t)\right]
\end{aligned}
$$

where $e_{f}(t)$ is the fault estimation error defined as follows:

$$
e_{f}(t)=f(t)-\hat{f}(t)
$$

Let us consider the following conditions:

$$
\begin{aligned}
& M_{1} A_{i}-L_{i} C-N_{i}+N_{i} M_{2} C=0 \\
& N_{i} M_{2}-L_{i}=K_{i} \\
& M_{1} A_{i}+K_{i} C=N_{i} \\
& M_{1} B_{i}-G_{i}=0 \\
& M_{1} F_{i}-H_{i}=0
\end{aligned}
$$

Therefore, by taking into account (16)-(20), the state estimation error dynamic (14) becomes:

$$
\dot{e}(t)=\sum_{i=1}^{h} \rho_{i}(\theta(t))\left[N_{i} e(t)+H_{i} e_{f}(t)\right]
$$

We assume that the fault $f(t)$ has a slow variation such that $\dot{f}(t) \simeq 0$. Thus, the fault estimation error dynamic can be expressed as:

$$
\dot{e}_{f}(t)=-\dot{\hat{f}}(t)=-\sum_{i=1}^{h} \rho_{i}(\theta(t)) \phi_{i}(y(t)-\hat{y}(t))
$$

Then, equations (21) and (22) can be combined in an augmented system as follows:

$$
\dot{\bar{e}}(t)=\sum_{i=1}^{h} \rho_{i}(\theta(t))\left(\bar{A}_{i}+\bar{K}_{i} \bar{C}\right) \bar{e}(t)
$$

where $\bar{e}(t)=\left(\begin{array}{c}e(t) \\ e_{f}(t)\end{array}\right) ; \bar{A}_{i}=\left(\begin{array}{cc}M_{1} A_{i} & M_{1} F_{i} \\ 0 & 0\end{array}\right)$;

$\bar{K}_{i}=\left(\begin{array}{c}K_{i} \\ \phi_{i}\end{array}\right)$ and $\bar{C}=\left(\begin{array}{ll}C & 0\end{array}\right)$.

The system dynamics (23) can be stabilized by choosing the gain $\bar{K}_{i}$ thanks to the detectability of each pair $\left(\bar{A}_{i} ; \bar{C}\right)$, $\forall i=1, \ldots h$.

Theorem 1: Let consider the polytopic LPV system (5) and the Polytopic Proportional Integral Observer (6). The augmented system (23) converges exponentially if there exists a symetric and positive definite matrix $P$, matrices $Q_{i}=P \bar{K}_{i}$, $K_{i}$ and $\phi_{i}$, and a positive scalar $\alpha$ for all $\rho(\theta(t)) \in \Omega$ such that:

$$
\bar{A}_{i}^{T} P+P \bar{A}_{i}+\bar{C}^{T} Q_{i}^{T}+Q_{i} \bar{C}+2 \alpha P \prec 0 ; \forall i=1, \cdots, h
$$

where

$$
\begin{gathered}
\bar{A}_{i}=\left(\begin{array}{cc}
M_{1} A_{i} & M_{1} F_{i} \\
0 & 0
\end{array}\right) \\
\bar{K}_{i}=\left(\begin{array}{c}
K_{i} \\
\phi_{i}
\end{array}\right)
\end{gathered}
$$


and

$$
\bar{C}=\left(\begin{array}{ll}
C & 0
\end{array}\right)
$$

The observer gains are given by:

$$
\begin{gathered}
N_{i}=M_{1} A_{i}+K_{i} C \\
L_{i}=N_{i} M_{2}-K_{i} \\
G_{i}=M_{1} B_{i}
\end{gathered}
$$

and

$$
H_{i}=M_{1} F_{i}
$$

\section{Proof 1:}

Let consider the quadratic Lyapunov function represented by the following form:

$$
V(\bar{e}(t))=\bar{e}(t)^{T} P \bar{e}(t)
$$

where $P=P^{T}>0$ and he convergence of the augmented system (23) is ensured if there exists $P=P^{T}>0$ such that:

$$
\dot{V}(\bar{e}(t))+2 \alpha V(\bar{e}(t)) \prec 0
$$

where $\alpha$ is the decay rate of the solution of the equation (33) described as:

$$
V(\bar{e}(t)) \leq V(0) \mathrm{e}^{-2 \alpha t} ; \forall t \geq 0
$$

Consider $V(\bar{e}(t)$ is bounded as follows:

$$
\lambda_{\min }(P)\|\bar{e}(t)\|^{2} \leq V(\bar{e}(t)) \leq \lambda_{\max }(P)\|\bar{e}(t)\|^{2}
$$

Therefore, the norm of the estimation error can be bounded by:

$$
\|\bar{e}(t)\| \leq \sqrt{\frac{\lambda_{\min }(P)}{\lambda_{\max }(P)}} \mathrm{e}^{-\alpha t}\|\bar{e}(0)\| ; \forall t \geq 0
$$

The dynamic of the quadratic Lyapunov function (32) is given by:

$$
\dot{V}(\bar{e}(t))=\dot{\bar{e}}(t)^{T} P \bar{e}(t)+\bar{e}(t)^{T} P \dot{\bar{e}}(t)
$$

By taking into account the augmented dynamic equation error (23), the equation (37) becomes:

$$
\begin{aligned}
\dot{V}(\bar{e}(t))= & \bar{e}(t)^{T} \sum_{i=1}^{h} \rho_{i}(\theta(t))\left[\left(\bar{A}_{i}+\bar{K}_{i} \bar{C}\right)^{T} P\right. \\
& \left.+P\left(\bar{A}_{i}+\bar{K}_{i} \bar{C}\right)\right] \bar{e}(t)
\end{aligned}
$$

Thus, the inequality (33) can be rewritten by:

$$
\begin{aligned}
& \dot{V}(\bar{e}(t))+2 \alpha V(\bar{e}(t))=\bar{e}(t)^{T} \sum_{i=1}^{h} \rho_{i}(\theta(t))\left[\left(\bar{A}_{i}+\bar{K}_{i} \bar{C}\right)^{T} P\right. \\
& \left.+P\left(\bar{A}_{i}+\bar{K}_{i} \bar{C}\right)+2 \alpha P\right] \bar{e}(t) \prec 0
\end{aligned}
$$

Then, the inequalities (39) are verified $\forall i \in\{1, \ldots, h\}$ if there exist a symmetrical and definite positive matrix $P$ and a matrices $Q_{i}=P \bar{K}_{i}$ such that the following LMIs hold true:

$$
\bar{A}_{i}^{T} P+P \bar{A}_{i}+\bar{C}^{T} Q_{i}{ }^{T}+Q_{i} \bar{C}+2 \alpha P \prec 0 ; \forall i=1, \cdots, h
$$

\section{B. Fault Detection and Diagnosis}

The difference between the roles of the Fault Detection and Isolation FDI and the Fault Detection and Diagnosis FDD is clarified here to avoid the confusion since terms "Isolation" and "Identification" share the same initials correspond to different functional cases. The FDI aims to locate and isolate faulty components in the system. The FDD, however in intended to know the detailed attributes of detected faults e.g faults severity and faults identification is required in FDD [6]. In this section, we proceed the technique of FDD using a polytopic PI observer in order to detect, isolate and identify the actuator faults for the LPV systems. To solve the FDD problem, we propose an algorithm composed by three steps:

1) Fault Detection: In the present study, a bank of polytopic PI observer is designed to generate residuals able to detect actuator faults. The residual generator can be considered as a polytopic PI observer adapted as follows [19]:

$$
\left\{\begin{array}{l}
\dot{z}(t)=\sum_{i=1}^{h} \rho_{i}(\theta(t))\left[N_{i} z(t)+G_{i} u(t)+L_{i} y(t)+H_{i} \hat{f}(t)\right] \\
\hat{x}(t)=z(t)+M_{2} y(t) \\
r(t)=y(t)-\hat{y}(t) \\
\dot{\hat{f}}(t)=\sum_{i=1}^{h} \rho_{i}(\theta(t)) \phi_{i}(y(t)-\hat{y}(t))
\end{array}\right.
$$

where $r(t)$ is the residual signal generated by the difference between measured outputs and estimated outputs. The exponential convergence of the augmented error estimation of the residual generator (41) is guaranteed if there exits a symetric and positive definite matrix $P$, matrices $Q_{i}=P \bar{K}_{i}$ and a positive scalar $\alpha$ for all $\rho(\theta(t)) \in \Omega$ such that the LMI (24) is verified (see Theorem1). The matrices $K_{i}$ and $\phi_{i}$ are given by the result of LMI solving (24). In addition, the matrices $N_{i}, L_{i}$, $G_{i}$ and $H_{i}$ are given by the constraints (28)-(31). Therefore, the proposed residual generator is able to detect faults estimate states at same time.

2) Fault Isolation: The idea is to make each residual sensitive to only one actuator fault and insensitive to all others faults [18].

We assume that, for each actuator fault $f_{j(t)}$, the polytopic LPV system is represented by the following state space:

$$
\left\{\begin{array}{l}
\dot{x}(t)=\sum_{i=1}^{h} \rho_{i}(\theta(t))\left[A_{i} x(t)+B_{i} u(t)+F_{i}^{j} f_{j}(t)\right] \\
y(t)=C x(t)
\end{array}\right.
$$

where $F_{i}^{j}$ is the fault distribution matrix of appropriate size for the $j^{\text {th }}$ actuator fault $f_{j}(t)$ with $j \in[1, \ldots, q]$. Then, we consider a bank of $q$ Polytopic PI Observer (PPIO) based residual generators designed by:

$$
\left\{\begin{array}{l}
\dot{z}_{j}(t)=\sum_{i=1}^{h} \rho_{i}(\theta(t))\left[N_{i}{ }^{j} z_{j}(t)+G_{i}{ }^{j} u(t)+L_{i}{ }^{j} y(t)\right. \\
\left.+H_{i}{ }^{j} \hat{f}_{j}(t)\right] \\
\hat{x}(t)=z_{j}(t)+M_{2} y(t) \\
r_{j}(t)=y(t)-\hat{y}(t) \\
\dot{\hat{f}}(t)=\sum_{i=1}^{h} \rho_{i}(\theta(t)) \phi_{i}{ }^{j}(y(t)-\hat{y}(t)) \\
\text { forj } \in[1, \ldots, q]
\end{array}\right.
$$


The matrices $\phi_{i}^{j}$ may be deduced by solving the LMI (24). Then, the matrices $N_{i}^{j}, L_{i}^{j}, G_{i}^{j}$ and $H_{i}^{j}$ can be computed by using the constraints (28)-(31).

Since, the probability to get a system affected by two or more faults at the same time [8], each residual vector $r_{j}(t)$ generated by the $j^{\text {th }}$ (PPIO) can be used to isolate a single fault at every moment.

3) Fault Estimation: In order to estimate the actuator fault, we use the designed (PPIO) that is able to estimate the states vector and the fault $f(t)$ at same time. Taking into account the equation of $\dot{\hat{f}}(t)$ in the (PPIO) structure (6), we can easily compute the estimated fault magnitude $\hat{f}(t)$.

\section{Application on a VAn de Vusse REACTOR}

In this section, a Van de Vusse reactor is used to illustrate the proposed method of fault detection and diagnosis FDD. A Van de Vusse reactor is known to be a highly nonlinear process. For this, it have been used by many researchers as a benchmark problem [12], [17]. In this reactor, a product $\mathrm{A}$ is converted to the desired product $\mathrm{B}$ in an isothermal continuous stirred tank reactor CSTR which has exothermic reaction instability with a prolonged cooling jacket temperature above $305 \mathrm{~K}$ as it is illustrated in the following figure: The product

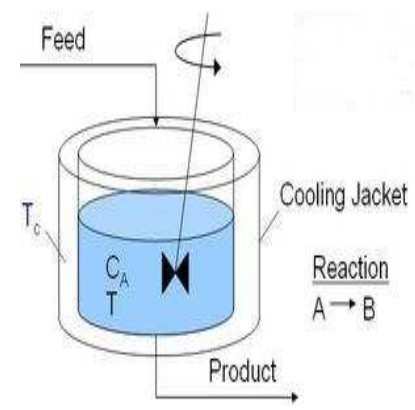

Fig. 1. Continuous stirred tank reactor CSTR

$\mathrm{B}$ is converted also to a product $\mathrm{C}$. In addition to this consecutive reaction, a high order parallel reaction occurs and A is converted to product D [12] as it is shown in the following reactions:

$$
\begin{gathered}
A \stackrel{k_{1}}{\rightarrow} B \stackrel{k_{2}}{\rightarrow} C \\
2 A \stackrel{k_{3}}{\rightarrow} D
\end{gathered}
$$

Consider the following dynamics of CSTR for the Van de Vusse reactor:

$$
\left\{\begin{array}{l}
\frac{d C_{A}}{d t}=-k_{1} C_{A}-k_{3} C_{A}^{2}+\left(C_{A f}-C_{A}\right) \frac{F_{u}}{V} \\
\frac{d C_{B}}{d t}=k_{1} C_{A}-k_{2} C_{B}+\left(-C_{B}\right) \frac{F_{u}}{V}
\end{array}\right.
$$

where $C_{A}, C_{B}$ and $C_{A f}$ are respectively the concentration of the reactant $A$ inside the reactor $(\mathrm{mol} / \mathrm{L})$, the concentration of the product B in the CSTR output stream (mol/L) and the concentration of the product $\mathrm{A}$ at the operating point. $F_{u}$ is the input flow rate to the rector $(\mathrm{L} / \mathrm{h})$ and $V$ is the constant volume of the CSTR (L). The kinetic parameters are chosen to be $k_{1}=100 h^{-1}, k_{2}=50 h^{-1}, k_{3}=10 h^{-1}, C_{A f}=10 \mathrm{~mol} / \mathrm{h}$ and $V=1 L$ as in [12].

Let consider that the Van de Vusse reactor is affected by an

additive actuator fault $f(t)$ and with the two following states $x_{1}(t)=C_{A}$ and $x_{2}(t)=C_{B}$ such that it becomes modeled by the following nonlinear representation:

$$
\left\{\begin{array}{l}
\dot{x}_{1}(t)=-k_{1} x_{1}(t)-k_{3} x_{1}^{2}(t)+\left(C_{A f}-x_{1}(t)\right)[u(t)+f(t)] \\
\dot{x}_{2}(t)=k_{1} x_{1}(t)-k_{2} x_{2}(t)+\left(-x_{2}(t)\right)[u(t)+f(t)] \\
y_{1}(t)=x_{1}(t) \\
y_{2}(t)=x_{2}(t)
\end{array}\right.
$$

where $x(t)=\left(\begin{array}{ll}x_{1}(t) & x_{2}(t)\end{array}\right)^{T}$ is the state vector,

$y(t)=\left(\begin{array}{ll}y_{1}(t) & y_{2}(t)\end{array}\right)^{T}$ is the output vector and $u(t)=\frac{F_{u}}{V}$ is the input.

Here, we put the previous nonlinear form in LPV form such that:

$$
\left\{\begin{array}{l}
\dot{x}(t)=A(\theta(t)) x(t)+B(\theta(t))[u(t)+f(t)] \\
y(t)=C x(t)
\end{array}\right.
$$

The matrices $A(\theta(t)), B(\theta(t))$ and $C$ are given by:

$$
\begin{aligned}
& A(\theta(t))=\left(\begin{array}{cc}
-k_{1}-k_{3} \theta_{1}(t) & 0 \\
k_{1} & -k_{2}
\end{array}\right) ; \\
& B(\theta(t))=\left(\begin{array}{c}
C_{A f}-\theta_{1}(t) \\
-\theta_{2}(t)
\end{array}\right) \text { and } C=\left(\begin{array}{ll}
1 & 0 \\
0 & 1
\end{array}\right)
\end{aligned}
$$

with the parameters $\theta_{1}(t)=x_{1}(t)$ and $\theta_{2}(t)=x_{2}(t)$ vary in a hypercube such that: $0.4 \leq \theta_{1}(t) \leq 1$ and $0.6 \leq \theta_{2}(t) \leq 1.1$. Finally, we can rewrite the proposed form (46) in the following polytopic LPV form:

$$
\left\{\begin{array}{l}
\dot{x}(t)=\sum_{i=1}^{4} \rho_{i}(\theta(t))\left[A_{i} x_{(t)}+B_{i}(u(t)+f(t))\right] \\
y(t)=C x(t)
\end{array}\right.
$$

where convex scheduling functions are defined as:

$$
\begin{aligned}
& \rho_{1}(\theta(t))=\frac{\theta_{1}(t)-\theta_{1 \min }}{\theta_{1 \max }-\theta_{1 \min }} \frac{\theta_{2}(t)-\theta_{2 \min }}{\theta_{2 \max }-\theta_{2 \min }} \\
& \rho_{2}(\theta(t))=\frac{\theta_{1}(t)-\theta_{1 \min }}{\theta_{1 \max }-\theta_{1 \min }} \frac{\theta_{2 \max }-\theta_{2}(t)}{\theta_{2 \max }-\theta_{2 \min }} \\
& \rho_{3}(\theta(t))=\frac{\theta_{1 \max }-\theta_{1}(t)}{\theta_{1 \max }-\theta_{1 \min }} \frac{\theta_{2}(t)-\theta_{2 \min }}{\theta_{2 \max }-\theta_{2 \min }} \\
& \rho_{4}(\theta(t))=\frac{\theta_{1 \max }-\theta_{1}(t)}{\theta_{1 \max }-\theta_{1 \min }} \frac{\theta_{2 \max }-\theta_{2}(t)}{\theta_{2 \max }-\theta_{2 \min }}
\end{aligned}
$$

And the matrices are described as follows:

$$
\begin{aligned}
& A_{1}=A_{2}=\left(\begin{array}{cc}
-k_{1}-k_{3} \theta_{1 \mathrm{~min}} & 0 \\
k_{1} & -k_{2}
\end{array}\right) \text {; } \\
& A_{3}=A_{4}=\left(\begin{array}{cc}
-k_{1}-k_{3} \theta_{1 \max } & 0 \\
k_{1} & -k_{2}
\end{array}\right) \text {; } \\
& B_{1}=\left(\begin{array}{c}
C_{A f}-\theta_{1 \min } \\
-\theta_{2 \min }
\end{array}\right) ; B_{2}=\left(\begin{array}{c}
C_{A f}-\theta_{1 \min } \\
-\theta_{2 \max }
\end{array}\right) ; \\
& B_{3}=\left(\begin{array}{c}
C_{A f}-\theta_{1 \max } \\
-\theta_{2 \min }
\end{array}\right) \text { and } B_{4}=\left(\begin{array}{c}
C_{A f}-\theta_{1 \max } \\
-\theta_{2 \max }
\end{array}\right) \text {. }
\end{aligned}
$$

\section{A. PPIO observer Design}

The solving of the LMI (24) drives to compute the unknown matrices of Polytopic PI Observer (6) by using the conditions (28)-(31). For $\alpha=107$, we obtain the matrices $N_{i}, L_{i}, G_{i}, H_{i}$ and $\phi_{i}(\forall i=1, \ldots, 4)$ which are used in Simulation.

In order to simulate the obtained results, the input flow rate 
$F_{u}$ is considered variable such that the input signal $u(t)$ will be expressed by:

$u(t)=\left\{\begin{array}{cc}6+\sin (0.07 t) & \text { for } \cdot t \succeq 0 \\ 0 & \text { elsewhere }\end{array}\right.$

and the fault is assumed as a rectangular signal such that: $f(t)=\left\{\begin{array}{cc}1.5 & \text { for } 10 \leq t \leq 15 \\ 0 & \text { elsewhere }\end{array}\right.$.

The above input $u(t)$ and fault $f(t)$ are applied on this studied reactor, whose states and their estimations given by the observer PPIO, are shown in the following figures:

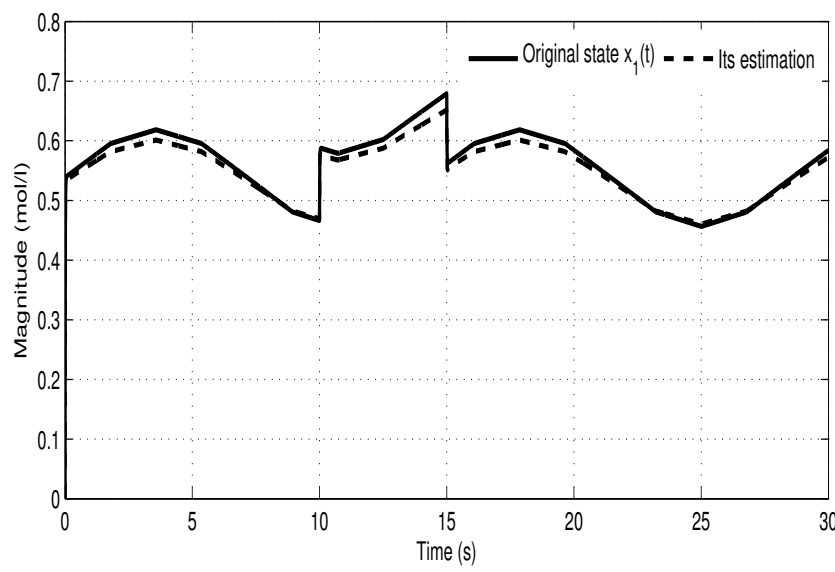

Fig. 2. Comparison between the original state $x_{1}(t)$ and its estimation $\hat{x}_{1}(t)$

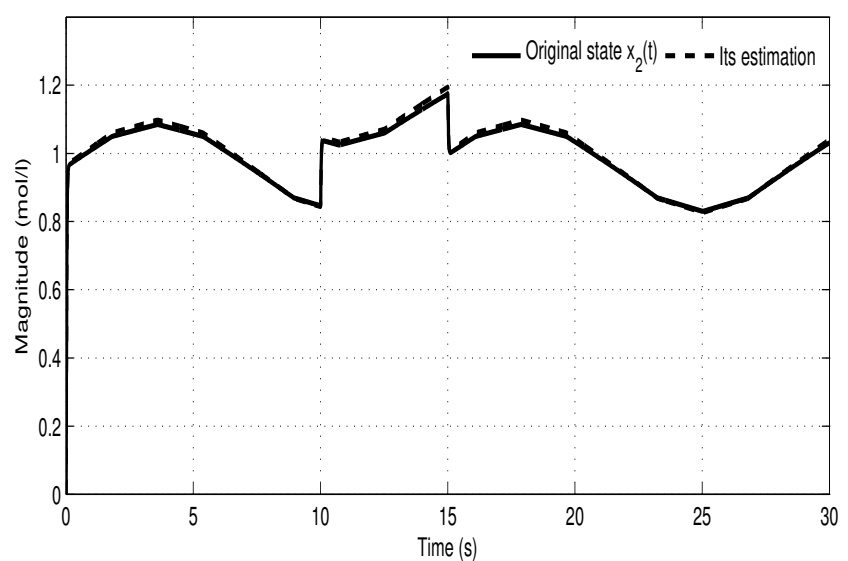

Fig. 3. Comparison between the original state $x_{2}(t)$ and its estimation $\hat{x}_{2}(t)$

Seeing these two figures (Fig.2 and Fig.3), we notice that dice the moment zero, the reals concentrations of the product A and the product B are practically similar to their estimated. This one shows the effectiveness of the proposed PPIO observer for the states estimation.

\section{B. Fault Detection and Diagnosis}

In this subsection, we consider that the polytopic LPV system (47) is affected by the above actuator fault $f(t)$. Besides, we consider a PPIO observer that able to generate the residual signal $r(t)$ illustrated by the following figure:

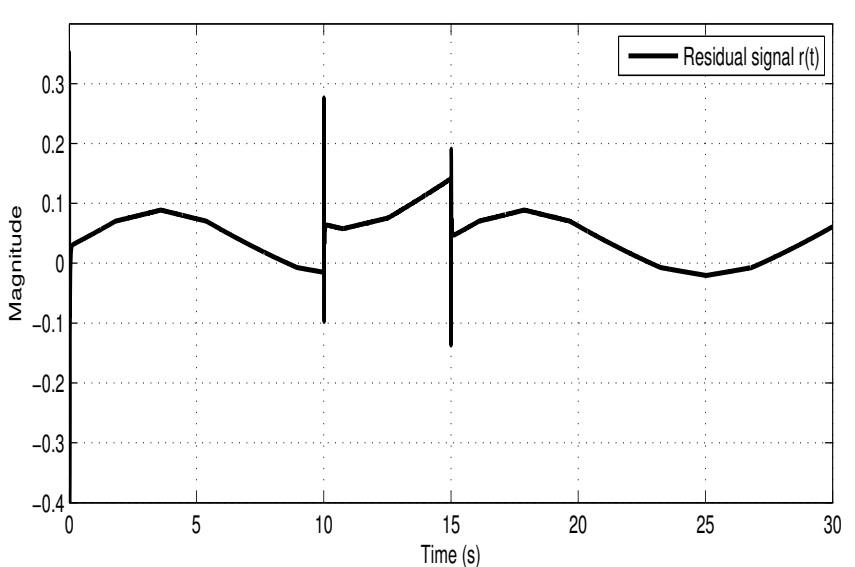

Fig. 4. The residual signal $r(t)$

This figure (Fig.4) shows that the proposed PPIO observer us allows to detect and isolate faults. It is clear that the fault affects slightly on the generated residual signal $r(t)$ which seems, in this case, still close to zero.

Finally, in the objective to estimate the actuator fault $f(t)$, we use the proposed PPIO observer structure (6) so as to calculate the magnitude fault which is shown as follows:

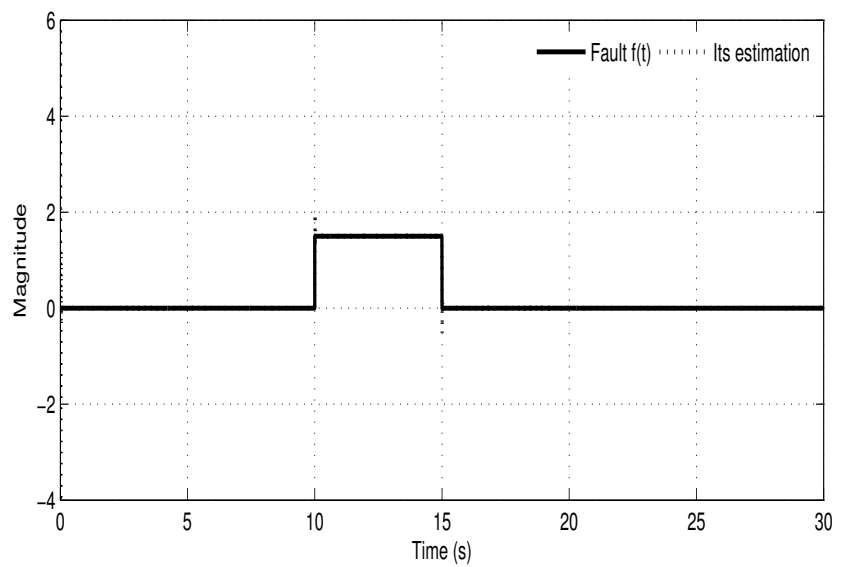

Fig. 5. Comparison between the actuator fault $f(t)$ and its estimation $\hat{f}(t)$

The figure (Fig.5) proves that the designed PPIO observer provides a very accurate estimated fault compared to the real actuator fault.

These results show that the use of a PPIO observer is a very good choice for the fault detection and diagnostic FDD of the polytopic LPV systems.

\section{CONClusion}

In this paper, the problem of faults detection and diagnosis FDD for a polytopic LPV systems was dealt. The proposed 
method consist to design a Polytopic Proportional Integral Observer (PPIO) that provides the estimated states. Besides, the proposed observer has been used to detect and isolate and estimate actuators faults. The (PPIO) gains are obtained by solving the linear matrices inequalities (LMIs). The developed FDD method for polytopic LPV systems has been applied into a Van de Vusse reactor in the objective to show its efficiency.

\section{REFERENCES}

[1] M. Adam-Medina, M. Rodrigues, D. Theilliol and H. Jamouli, Fault diagnosis in nonlinear systems through an adaptative filter under a convex set representation. European Control Conference, ECC 2003, Cambridge: Royaume-Uni 2003.

[2] A. Aguilera-Gonzalez, D. Theilliol, M. Adam-Medina, C.M. AstorgaZaragoza and M. Rodrigues, Sensor Fault and Unknown Input Estimation Based on Proportional Integral Observer Applied to LPV Descriptor Systems. 8th IFAC Symposium on Fault Detection, Supervision and Safety of Technical Processes, SAFEPROCESS 2012, Mexico City: Mexique 2012.

[3] C. M. Astorga-Zaragoza, D. Theilliol, J. C. Ponsart and M. Rodrigues, Fault diagnosis for a class of Descriptor Linear Parameter Varying systems. International Journal of Adaptive Control and Signal Processing 2011 Early View DOI: 10.1002/acs.1259.

[4] C. M. Astorga-Zaragoza, D. Theilliol, J. C. Ponsart and M. Rodrigues, Sensor fault diagnosis for a class of LPV descriptor systems. 7th Workshop on Advanced Control and Diagnosis, Poland 2009.

[5] M. Darouach and M. Boutayeb, Design of Observers for Descriptor Systems. IEEE transactions on Automatic Control, vol 40, 1995, pp 1323-1327.

[6] S. X. Ding, Model-Based Fault Diagnosis Techniques Design Schemes, Algorithms and Tools. Advances in Industrial Control Springer 2013.

[7] P. Gohinet P. Apkarian and M. Chiloli, Affine Parameter-Dependent Lyapunov Functions for Real Parainetric Uncertainty. Poceedings of 33rd Conference on Decision and Control Lake Buena Vista - FL December 1994.

[8] H. Hamdi, M. Rodrigues, C. Mechmeche, D. Theilliol and N. BenHadj Braiek, Fault detection and isolation in linear pa-rameter-varying descriptor systems via proportional integral observer. International journal of adaptive control and sig-nal processing, DOI: 10.1002/acs.1260, 2011.

[9] H. Hamdi, M. Rodrigues, C. Mechmeche and N. BenHadj Braiek, Robust Fault Detection and Estimation for Descriptor Systems Based on MultiModels Concept. International Journal of Control - Automation and Systems, Vol. 10, no. 6, pp 1260-1266, December 2012.

[10] H. Hamdi, M. Rodrigues, C. Mechemeche, D. Theilliol and N. Benhadj Braiek, State Estimation for Polytopic LPV Descriptor Systems: Application to Fault Diagnosis. the 7th IFAC Symposium on Fault Detection, Supervision and Safety of Technical Processes, Barcelona, Spain, June 30 - July 3, 2009.

[11] D. Henry and A. Zolghadri, Robust fault diagnosis in uncertain Linear Paramet er,-Varying systems. IEEE International Conference on Systems, Man and Cybernetics 2004.

[12] H. Kashiwagi, L. Rong, Identification of Volterra kernels of nonlinear Van de Vusse reactor. International Journal of Control Automation and Systems. 4(2):109-113, 2002.

[13] D.Koenig and S. Mammar, Design of Proportional-Integral Observer for Unknown Input Descriptor Systems. IEEE transactions on automatic control, december 2002, vol 47, N12. 13.

[14] D. Koenig, Unknown Input Proportional Multiple-Integral Observer Design for Linear Descriptor Systems: Application to State and Fault Estimation. IEEE Transactions on Automatic control February 2005; Vol 50, N2, pp 212-217.

[15] B. Marx, D. Koenig and J. Ragot, Design of observers for TakagiSugeno descriptor systems with unknown inputs and application to fault diagnosis. IET control theory applica- tions, vol.1, no.5, pp 1487-1495, 2007.

[16] C. Mechmeche, H. Hamdi, M. Rodrigues, N. BenHadjBraiek, State and Unknown Inputs Estimations for Multi-Models Descriptor Systems. American Journal of Computational and Applied Mathematics 2, 3 (2012) pages 86-93, DOI: 10.5923/j.ajcam.20120203.04

[17] H. Perez, B. Ogunnaike, and S. Devasia, Output tracking between operating points for nonlinear processes: Van de Vusse example. IEEE Transactions on Control Systems Technology. 8(2):200-211, 2000,
[18] M. Rodrigues, M. Adam-Medina, D.Theilliol and D.Sauter, Fault Diagnosis on industrial systems based on a multiple model approach. Symposium on automation in Mining, Mineral and Metal processing 2004.

[19] M. Rodrigues, H. Hamdi, D. Theilliol, C. Mechmeche and N. BenHadj Braiek, Actuator Faut Estimation based Adaptive Polytopic Observer for a Class of LPV Descriptor Systems. International Journal of Robust and Nonlinear Control, Int. J. Robust. Nonlinear Control 2014; 00:115. DOI: $10.1002 /$ rnc.

[20] M. Rodrigues, M. Sahnoun, D. Theilliol and J.C. Ponsart, Sensor Fault Detection and Isolation Filter for Polytopic LPV Systems: A Winding Machine Application. Journal of Process Control 23, 6 (2013) 805-816" DOI : 10.1016/j.jprocont.2013.04.002.

[21] M. Rodrigues, D. Theilliol and D. Sauter, Fault diagnosis for nonlinear system based on multimodel reprsentation: a robust polytopic unknown input obsever synthesis. 44th IEEE Conference on Decision and Control and European Control Conference ECC, Espagne -2005.

[22] Z. Wang, M. Rodrigues, D. Theilliol, Y. Shen, Actuator fault estimation observer design for discrete-time linear parameter-varying descriptor systems. International Journal of Adaptive Control and Signal Processing - 2014 - 1-9 DOI: 10.1002/acs.2469. 